\title{
Clinical Characteristics and Treatment Outcome of Paediatric Rhabdomyosarcoma; A Retrospective Review
}

\section{Raheela Mansoor, Zunaira Shaukat, Najma Shaheen, Saliha Sarfraz, Komal Seher}

Department of Paediatric Oncology, Shaukat Khanum Memorial Cancer Hospital and Research Centre, Lahore, Punjab, Pakistan

Received: 13 December 2020/Accepted: 28 May 2021

C. Open access Correspondence:

Zunaira Shaukat, Department of Paediatric Oncology, Shaukat Khanum Memorial Cancer Hospital and Research Centre, Lahore, Punjab, Pakistan.

E-mail: zsaq0028@hotmail.com

Citation: Mansoor R, Shaukat Z, Shaheen N, Sarfraz S, Seher K. Clinical Characteristics and Treatment Outcome of Paediatric Rhabdomyosarcoma; A Retrospective Review. J Cancer Allied Spec [Internet]. 2021 May 31;7(2). e1002957. https://doi.org/10.37029/jcas. v7i2.393

Copyright: (c) 2021 Mansoor, et al.This is an open access article distributed under the terms of the Creative Commons Attribution License, which permits unrestricted use, distribution, and reproduction in any medium, provided the original author and source are credited.

Funding: The authors received no financial support for the research, authorship and/or publication of this article.

Competing interest: Nil.

\begin{abstract}
Introduction: Rhabdomyosarcoma (RMS) is the most common soft tissue sarcoma in children. This paper aimed to assess the stage, site and treatment outcome among RMS patients. Materials and Methods: A retrospective chart review was completed from January 2011 to December 2017 of patients that presented to the Department of Paediatric Oncology, Shaukat Khanum Memorial Cancer Hospital, Lahore, Pakistan, for the management of RMS. Data collection included clinical characteristics, staging, grouping, risk stratification, treatment plan, radiotherapy doses and treatment outcome. Results: Among 24 subjects, there were a total of 13 (54.2\%) males and 11 (45.8\%) females. The median age at the time of diagnosis was 2.5 years (range: $0.75-17$ years). The majority of the subjects (91.7\%) were $<10$ years of age. The median follow-up time was 0.6 years. According to the Children's Oncology Group Classification, 4 (16.7\%) subjects were classified as low risk, 14 (58.3\%) subjects were rated as intermediate risk and 6 (0.25\%) subjects were stratified as high risk. The most common primary tumour site was genitourinary (62.5\%) and abdomen/retroperitoneal (20.8\%) regions. At the time of analysis, nine (37.5\%) subjects had died because of the disease, 12 (50\%) were alive with no evidence of disease and one subject had a recurrence of disease and was alive. One subject had abandoned the therapy and another was lost to follow-up. Conclusion: Patients with RMS presented at the late stages of the disease and it most frequently affected genitourinary and abdomen or retroperitoneal areas. Overall, RMS was found to have a poor outcome to therapy.
\end{abstract}

Key words: Alveolar, embryonal, low-middle income country, rhabdomyosarcoma, treatment

\section{Introduction}

Rhabdomyosarcoma (RMS) is the most common soft tissue sarcoma in children, with approximately 350 cases diagnosed per year in the United States. ${ }^{[1]}$ The two major histological types are embryonal RMS (ERMS) and alveolar RMS (ARMS). There is a disparity between the tumour site, histology and clinical behaviour of RMS.[2] Paediatric RMS with embryonal histology and a location in orbit, head and neck, or genitourinary tract has a significantly better prognosis than 
the tumours with alveolar histology that arise in

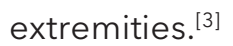

Treatmentstrategies include systemic chemotherapy along with surgery and/or radiotherapy as local control options. Multimodality treatment has resulted in 5 -year survival of approximately $67 \% .{ }^{[3-5]}$ There are limited data on the outcome of RMS from developing countries. ${ }^{[6,7]}$ Data from Pakistan are scarce with poor outcomes. Bhurgri et al. reported a $10 \% 5$-year survival in a hundred patients studied. ${ }^{[8]}$ Issues contributing to dismal results in this part of the world include delay in seeking medical help, lack of surgical expertise for local disease control, failure to deliver chemotherapy in a timely fashion and family compliance..$^{[9]}$

The purpose of this study was to assess the stage, site and treatment outcome among patients presenting with RMS in a tertiary care cancer hospital in Pakistan.

\section{Materials and Methods}

The Institutional Review Board of the hospital approved this analysis (EXMPT-19-02-18-01). In this study, a 7-year retrospective chart review of 24 RMS patient records from January 2011 to December 2017 who were younger than 18-years of age was conducted. All other patients registered in the same period were excluded from the analysis. Data collection included clinical characteristics, staging, grouping, risk stratification, treatment plan, radiotherapy doses and outcome of children with RMS treated at the Shaukat Khanum Memorial Cancer Hospital and Research Centre (SKMCH) Lahore, Pakistan.

The study participants were risk-stratified using the Children's Oncology Group (COG) Classification [Figure 1]. ${ }^{[10,11]}$ The classification depends on both the pre-treatment staging system and the surgical and histopathologic grouping scheme. It takes into consideration the size, site, invasiveness and nodal status of the tumour. The grouping system is based on the gross and microscopic extent of the residual tumour with consideration of regional nodes.
The subjects with embryonal histology were classified as low risk (LR). They were further stratified as Stage I with a Group I, II, or III, or Stage II with a Group I or II. The subjects with non-metastatic alveolar histology with Stages I, II, or III and a Groups I, II and III and unreacted embryonal tumour with an unfavourable site and Stage I or II with a Group III were considered to be at intermediate risk (IR). Similarly, all participants with Stage IV metastatic tumours with a Group IV were regarded as high risk (HR).

The participants underwent neoadjuvant chemotherapy followed by local control through surgery and/or radiation therapy (RT). Baseline staging and then re-evaluation at week 9 were done using computed tomography thorax and magnetic resonance imaging of the primary site. Four patients had chemotherapy as per the European Soft Tissue Sarcoma Study Group(EpSSG) protocol.[12,13] However, given the scarcity of bed availability for chemotherapy administration, the physicians decided to switch to the COG protocols given the ease of administration of chemotherapy as an outpatient service. ${ }^{[14,15]}$ Chemotherapy agents involved were vincristine, actinomycin, cyclophosphamide (VAC), ifosfamide, etoposide (IE) and irinotecan. LR participants received treatment as per ARST 0331 with 22 weeks of therapy. ${ }^{[16]}$ The treatment regimen comprised four cycles of VAC, with a total cyclophosphamide cumulative dose of $4.8 \mathrm{~g} / \mathrm{m}^{2}$, followed by four cycles of vincristine and actinomycin (VA) D. The IR subjects received therapy as per ARST1431 with 42 weeks of VAC alternating with vincristine and irinotecan (VAC) $\mathrm{VI}$ ) and radiation starting at $13^{\text {th }}$ week. The HR group received treatment as perARST 0431 with compressed cycles of vincristine, doxorubicin, cyclophosphamide and IE added to the backbone of VACNI. ${ }^{[17,18]}$

Statistical analysis was done using Statistical Package for the Social Sciences version 2022.0 (SPSS Inc, Chicago, IL). The frequencies were calculated for quantitative variables using descriptive statistics.

\section{Results}

There were a total of 13 (54.2\%) males and $11(45.8 \%)$ females. The median age of diagnosis 


\begin{tabular}{|c|c|c|}
\hline Stage & Site & Risk Classification \\
\hline $\begin{array}{l}\text { Stage } \\
\text { 1. Favorable } \\
\text { 2. Unfavorable site, }<5 \mathrm{~cm} \text {, any size bladder or } \\
\text { prostate disease, or no evidence of nodal } \\
\text { disease } \\
\text { 3. Unfavorable site, }>5 \mathrm{~cm} \text {, or evidence of } \\
\text { nodal disease } \\
\text { 4. Metastatic disease }\end{array}$ & \begin{tabular}{l}
\multicolumn{1}{c}{ Favorable sites } \\
- Orbit \\
- Head and neck (not parameningeal area) \\
- Genitourinary (not bladder or prostate) \\
- Biliary tract or liver
\end{tabular} & \begin{tabular}{l}
\multicolumn{1}{c}{ Intermediate risk } \\
- ARMS non-metastatic Stage 1, 2 or 3: Group \\
I, II or III \\
- ERMS Stage 2 or $3:$ Group III \\
- ERMS Stage 4: Group IV and age $<10$ years
\end{tabular} \\
\hline
\end{tabular}

Figure 1: Rhabdomyosarcoma staging and risk stratification based on the Children's Oncology Group Classification ${ }^{[11]}$ (embryonal rhabdomyosarcoma and alveolar rhabdomyosarcoma)

was 2.5 years with an interquartile range (IQR) of 2 years ( $1-3$ years). Most of the subjects ( $n=22$; $91.7 \%$ ) were $<10$ years of age. The median followup time was 7-months with an IQR of 7-months (4-11 months). According to the COG classification, four $(16.7 \%)$ subjects were classified as LR, $14(58.3 \%)$ subjects were considered to be IR and six $(0.25 \%)$ participants were categorized as HR. There were two ( $8.3 \%)$ subjects that had the alveolar subtype. The primary sites of tumour were head and neck area $(n=4,16.7 \%)$, genitourinary region ( $n=15,62.5 \%)$ and abdomen/ retroperitoneal region ( $n=5,20.8 \%)$. Among the study participants, two participants had ARMS and the rest were diagnosed with ERMS.

At the time of analysis, nine (37.5\%) subjects had died because of the disease, $12(50 \%)$ subjects were alive with no evidence of disease and one participant was alive with evidence of disease. One participant had abandoned therapy and another was lost to follow-up [Table 1]. All subjects $(n=4)$ with LR disease were alive at the time of the last follow-up in the clinic. Three of these subjects underwent systemic chemotherapy consisting of VAC for four cycles, followed by VA for 12 weeks. On the contrary, one of these four subjects underwent chemotherapy with VAC and VA for eight cycles. Three subjects were treated with surgery for local control within the participants with LR disease, while one subject underwent both surgery and RT.

Out of the 14 subjects stratified as IR, seven (50\%) were alive at the last follow-up visit. For local control among the IR group, six subjects had radiation and surgery, four participants received radiation alone and two subjects had surgery alone. Local control could not be achieved (they did not undergo surgery or receive RT) in two subjects. Both of these participants died. Among the six subjects that got both surgery and radiation for 
Table 1: The demographic and tumor characteristics of the cohort

\begin{tabular}{|c|c|c|c|c|}
\hline Characteristics & All & LR & IR & HR \\
\hline \multicolumn{5}{|l|}{ Gender } \\
\hline Male & 13 & 2 & 7 & 4 \\
\hline Female & 11 & 2 & 7 & 2 \\
\hline \multicolumn{5}{|l|}{ Age (years) } \\
\hline Median & 2.5 & 3 & 2 & 2 \\
\hline Range & $0.9-17$ & $0.9-7$ & $1-16$ & $1-17$ \\
\hline \multicolumn{5}{|l|}{ Residence } \\
\hline Afghanistan & 3 & 0 & 1 & 2 \\
\hline Khyber Pakhtunkhwa & 10 & 1 & 8 & 1 \\
\hline Punjab & 10 & 3 & 5 & 2 \\
\hline Sindh & 1 & 0 & 0 & 1 \\
\hline \multicolumn{5}{|l|}{ Tumour Site } \\
\hline Favourable & 11 & 4 & 4 & 3 \\
\hline Unfavourable & 13 & 0 & 10 & 3 \\
\hline \multicolumn{5}{|l|}{ Histology } \\
\hline Embryonal & 22 & 4 & 13 & 5 \\
\hline Alveolar & 2 & 0 & 1 & 1 \\
\hline \multicolumn{5}{|l|}{ Group } \\
\hline 1 & 3 & 2 & 1 & 0 \\
\hline II & 0 & 0 & 0 & 0 \\
\hline III & 20 & 2 & 13 & 5 \\
\hline IV & 1 & 0 & 0 & 1 \\
\hline \multicolumn{5}{|l|}{ Staging } \\
\hline 1 & 9 & 4 & 3 & 2 \\
\hline 2 & 1 & 0 & 1 & 0 \\
\hline 3 & 13 & 0 & 10 & 3 \\
\hline 4 & 1 & 0 & 0 & 1 \\
\hline \multicolumn{5}{|l|}{ Local control } \\
\hline Surgery alone & 5 & 3 & 2 & $1^{\mathrm{a}}$ \\
\hline Radiation alone & 5 & 0 & $4 *$ & $1^{\mathrm{b}}$ \\
\hline Both & 10 & 1 & $6 * *$ & $3^{c}$ \\
\hline None & 4 & 0 & $2 * * *$ & $1^{\mathrm{d}}$ \\
\hline
\end{tabular}

*(1 lost follow-up), **(all died), ***(both died), ${ }^{\text {a }}$ (abandoned therapy), ${ }^{b}$ (alive with disease), ${ }^{c}\left(\right.$ Two of them died), ${ }^{\mathrm{d}}$ (progressed and died), LR: Low risk, HR: High risk, IR: Intermediate risk

local disease, four (66.7\%) participants died. Half of these died from the progression of the disease, while the other half succumbed to therapy-related complications. The two subjects that had surgery alone were alive and doing well at the time of the analysis. All subjects in the IR group received chemotherapy. Chemotherapy was provided as per the EpSSG-2005 protocol in two subjects, five subjects received VAC for 12 cycles followed by a combination of VA and VI cycles and seven subjects got the VAC and VI for seven cycles each.

Three subjects having HR disease were managed by surgery and radiation. At the time of data collection, only one of these three subjects was disease-free. One participant was from Afghanistan who, after undergoing surgery, abandoned the therapy due to domestic issues and was lost to the follow-up. Out of the two subjects with extensive abdominal disease, one was alive with the disease after chemotherapy and RT. At the same time, the other progressed and died before any local control measure. EpSSG-2005 HR protocol was used for two subjects, while three got the VACNI protocol. The most recently admitted child's chemotherapy was as per ARST0431.

Twenty participants were to receive RT according to their post-surgical group. Participants with ERMS Group I tumours were not given radiation. There were no participants with Group II disease in this cohort. Nine participants (45\%) among those with Group III disease $(n=20 ; 100 \%)$ died before or after getting palliative doses of RT for local control. Three participants declined RT for local control. The remaining eight subjects (40\%) got radiation of 45 Gray units to genitalia and 50.4 Gray units to abdominal-pelvic disease sites.

\section{Discussion}

This retrospective review reports a descriptive analysis of 24 children treated at a tertiary care cancer centre in a lower-middle-income country. The study aimed to assess the stage, site and treatment outcome among subjects with RMS. Most of the tumours were Stage 3 (54.2\%) at presentation. Embryonal histology was most prevalent ( $n=22,91.7 \%)$. By the last follow-up date, out of 24 subjects, 9 (37.5\%) had died because of disease, 12 (50\%) were alive with the disease in remission and one was alive with evidence of disease. One subject abandoned active treatment and another was lost to follow-up. 
Prior studies have identified two critical prognostic factors in RMS. These are tumour histology and age at the time of diagnosis. ${ }^{[13,14]}$ The mortality rates are high in patients with ARMS because of its aggressive and metastatic nature. Children $<10$ years of age are likely to have ERMS and those 10 years or older are likely to have ARMS.[13,14] Similarly, older age is associated with a low overall survival rate. ${ }^{[13,14]}$ In the present study, there were two subjects with ARMS histology. Both subjects were not alive at the time of analysis.

A study by Qureshi and Rizvi from Karachi, Pakistan found similar results to the present study. Most RMS participants had advanced disease and the embryonal subtype was the more prevalent histopathology. ${ }^{[19]}$ The exact reason for presenting at advanced stages of the disease in low-income or low-middle income countries was beyond the scope of the present study. However, it is likely to be associated with a lack of public awareness, denial of illness and a fragmented health-care system, which creates accessing healthcare facilities difficult. Furthermore, there are many obstacles to cancer management among the paediatric population in developing countries. ${ }^{[9]}$ These include but are not limited to delayed diagnosis, cost of travel and accommodation to tertiary care centres, family education and abandonment. ${ }^{[20]}$

There are several limitations in the present study. First, a small number of subjects were included in the study and the duration of follow-up was short. Due to this, 5-year and overall survival were not calculated. Second, within the small cohort, two subjects abandoned the therapy. Monetary and infrastructure constraints are key dilemmas for families seeking care. Among the lost subjects, one was from Afghanistan and the other was from Federally Administered Tribal Areas. Third, this was a retrospective analysis. Inherently, this research design is inclined to recall, reporting and observational bias. Nonetheless, in the present study, the data were extracted from the electronic hospital information system and correlated with patient visits to the hospital to reduce the risk of recall and reporting bias.
This article, to the best of our knowledge, is the first published data of RMS stratification and treatment outcomes from Pakistan. To have a favourable outcome in RMS, the patients must undergo correct stratification. Analysis of our data highlights the RMS variations in terms of age and clinical presentations in the population of Pakistan. In this analysis, the COG classification is used. However, current best practices suggest that oncologist should incorporate fusion status during the workup for stratification. ${ }^{[21]}$ In the present centre, we will be incorporating FOXO1 gene rearrangement fluorescence in situ hybridization test during the work-up of RMS.

Patients with RMS presented at the late stages of the disease and it most frequently affected genitourinary and abdomen or retroperitoneal areas. Overall, in comparison to other Asian countries, children with RMS from Pakistan have inferior outcomes.

\section{Acknowledgments}

We acknowledge our patients who help us learn from and care for them.

\section{References}

1. Pizzo PA, Poplack DG. Principles and Practice of Pediatric Oncology. $6^{\text {th }}$ ed. Philadelphia, PA: Lippincott Williams and Wilkins; 2011.

2. van der Graaf WT, Orbach D, Judson IR, Ferrari A. Soft tissue sarcomas in adolescents and young adults: A comparison with their paediatric and adult counterparts. Lancet Oncol 2017;18:e166-75.

3. Gatta G, Botta L, Rossi S, Aareleid T, Bielska-Lasota M, Clavel J, et al. Childhood cancer survival in Europe 1999-2007: Results of EUROCARE-5-a populationbased study. Lancet Oncol 2014;15:35-47.

4. Meza JL, Anderson J, Pappo AS, MeyerWH, Children's Oncology Group. Analysis of prognostic factors in patients with nonmetastatic rhabdomyosarcoma treated on Intergroup Rhabdomyosarcoma Studies III and IV: The children's oncology group. J Clin Oncol 2006;24:3844-51.

5. Stevens MC, Rey A, Bouvet N, Ellershaw C, Flamant F, Habrand JL, et al. Treatment of nonmetastatic rhabdomyosarcoma in childhood and adolescence: Third study of the international society of paediatric oncology-SIOP malignant mesenchymal tumour 89. J Clin Oncol 2005;23:2618-28. 
6. Thavaraj V, Kumar R, Dawar R, Gupta DK, Mohanti BK, Singh $R$, et al. Treatment outcome and survival of rhabdomyosarcoma (RMS) in children. Med Pediatr Oncol 2001;37:286.

7. Dua V, Yadav SP, Prakash A, Sachdeva A. Encouraging treatment outcomes of pediatric rhabdomyosarcoma: A developing world experience. Pediatr Hematol Oncol 2012;29:677-8.

8. Bhurgri $Y$, Bhurgri A, Puri R, Ashraf S, Qidwai A, Ashraf $\mathrm{K}$, et al. Rhabdomyosarcoma in Karachi 1998-2002. Asian Pac J Cancer Prev 2004; 5:284-90.

9. Antillon F, Castellanos M, Valverde P, LunaFineman S, Garrido C, Serrato T, et al. Treating pediatric soft tissue sarcomas in a country with limited resources: The experience of the Unidad Nacional de oncologia pediatrica in guatemala. Pediatr Blood Cancer 2008;51:760-4.

10. Raney RB, Maurer HM, Anderson JR, Andrassy RJ, Donaldson SS, Qualman SJ, et al. The intergroup rhabdomyosarcoma study group (IRSG): Major lessons from the IRS-I through IRS-IV studies as background for the current IRS-V treatment protocols. Sarcoma 2001;5:9-15.

11. Lawrence W Jr., Anderson JR, Gehan EA, Maurer $\mathrm{H}$. Pretreatment TNM staging of childhood rhabdomyosarcoma: A report of the intergroup rhabdomyosarcoma study group. Children's cancer study group. Pediatric oncology group. Cancer 1997;80:1165-70.

12. Rudzinski ER, Kelsey A, Vokuhl C, Linardic CM, Shipley J, Hettmer S, et al. Pathology of childhood rhabdomyosarcoma: A consensus opinion document from the Children's oncology group, European paediatric soft tissue sarcoma study group, and the cooperative weichteilsarkom studiengruppe. Pediatr Blood Cancer 2021;68:e28798.

13. Dagher R, Helman L. Rhabdomyosarcoma: An overview. Oncologist 1999;4:34-44.

14. Sultan I, Qaddoumi I, Yaser S, RodriguezGalindo C, Ferrari A. Comparing adult and pediatric rhabdomyosarcoma in the surveillance, epidemiology and end results program, 1973 to 2005: An analysis of 2, 600 patients. J Clin Oncol 2009;27:3391-7.

15. Malempati S, Hawkins DS. Rhabdomyosarcoma: Review of the children's oncology group (COG) soft- tissue sarcoma committee experience and rationale for current COG studies. Pediatr Blood Cancer 2012;59:5-10.

16. Walterhouse DO, Pappo AS, Meza JL, Breneman JC, Hayes-Jordan AA, Parham DM, et al. Shorterduration therapy using vincristine, dactinomycin, and lower-dose cyclophosphamide with or without radiotherapy for patients with newly diagnosed low-risk rhabdomyosarcoma: A report from the soft tissue sarcoma committee of the children's oncology group. J Clin Oncol 2014;32:3547-52.

17. Hawkins DS, Anderson JR, Mascarenhas L, McCowage GB, Rodeberg DA, Wolden SL, et al. Vincristine, dactinomycin, cyclophosphamide (VAC) versus VACN plus irinotecan (VI) for intermediate-risk rhabdomyosarcoma (IRRMS): A report from the children's oncology group soft tissue sarcoma committee. ASCO Meet Abs 2014;32 Suppl 15:10004.

18. Weigel BJ, Lyden E, Anderson JR, Meyer WH, Parham DM, Rodeberg DA, et al. Intensive multiagent therapy, including dose-compressed cycles of ifosfamide/etoposide and vincristine/doxorubicin/ cyclophosphamide, irinotecan, and radiation in patients with high-risk rhabdomyosarcoma: A report from the children's oncology group. J Clin Oncol 2016;34:117-22.

19. Qureshi BM, Rizvi A. Prognostic factors and their influence on therapeutic outcomes in children and adolescents with parameningeal rhabdomyosarcoma: A multicenter study from Pakistan. Pediatr Hematol Oncol J 2021;6:6-11.

20. Arora RS, Eden T, Pizer B. The problem of treatment abandonment in children from developing countries with cancer. Pediatr Blood Cancer 2007;49:941-6.

21. Rudzinski ER, Anderson JR, Chi YY, Gastier-Foster JM, Astbury C, Barr FG, et al. Histology, fusion status, and outcome in metastatic rhabdomyosarcoma: A report from the children's oncology group. Pediatr Blood Cancer 2017;64:e26645.

\section{Authorship Contributions}

Conceived and designed the analysis; $\mathrm{RH}$ and NS. Collected the data; RH, ZS, SS and KS. Contributed data or analysis tools; ZS, NS, SS and KS. Performed the analysis; ZS, NS and SS. Wrote the paper; RH, ZS, NS, SS and KS. 